\title{
Blutkrankheiten und Blutdiagnostik.
}

\section{Lehrbuch}

der morphologischen Hämatologie.

Dr. med. Otto Naegeli,

Privatdozent an der Univęrsitat Zürich. Erste Hälfte.

ilit 13 Figuren im Text und 4 farbigen Tafeln.

Roy. 8. 1907 . geh. 7 ..

Die zweite Hälfte erscheint im Herbst 1907.

\section{Lehrbuch der Mathèmatik}

für Studierende der Naturwissenschaften und der Technik.

Einführung in die Differential- und Integralrechnung und in die analytische Geometrie.

Von

Dr. Georg Scheffers,

o. Professor an der Tecbnischen Bochschule Cnarlottenburg.

mit 344 Flguren.

Lex. 8. 1905. geh. $16 \%$, geb. in Ganzleinen $17,50 \%$.

Das Buch ist für diejenigen bestimmt, denen die Mathematik nur eine Hilfswissenschaft ist, und die sich durch Selbststudium mit den Begriffen und Methoden der höheren Mathematik vertraut machen. wollen. Es gebt von dem denkbar geringsten Mabe von Vorkenntnissen aus. Der Leser braucht nur im Buchstabenrechnen, in der Auflösung von Gleichungen ersten Grades mit einer Unbekannten und in der niederen Geometrie bewandert zu sein. Den, der es aufmerksam studiert, fördert es soweit, daB er die in seinem Forschungagebiet auftretenden Anwendungen der Mathematik versteht.

\section{Suggestion und Hypnotismus}

in der Völkerpsychologle.

Von

Dr. med. Otto Stoll,

o. Profegser der Geographie und Ethnologie an der Univeraitst Zarich.

Zweite, umgearbeitete und vermehrte Auflage.

Lex. 8. 1904. geh. $16 \boldsymbol{N}$, geb. in Halbfranz $18 \boldsymbol{N} 50$ \%

In diesem ausgezeichneten Werke werden zunächst die abnormen BewuBtseinszustände, deren Vorhandensein sich über die ganze Erde verbreitet im religiösen Leben aller Völker nachweisen läBt: die Erscheinungen der Ekstase, der Besessenheit, der einfachen Visionen. (und Gehörstänschungen), die Anăsthesie bei Martern, die Wachsuggestion bei den Zaubermanipulationen und die.sug. gestiven Heilwirkungen, also das ganze Gebiet der Wundererscheinungen in der Religion und die Wunderleistungen der Priester bei den tiefer wie den höher stehenden Völkers, psychologisch erklärt.

Sodann werden die neuzeitlichen Wachsuggestionen des politischen und wirtschaftlichen, des wissenschaftlichen und des künstlerischen Lebens bei denwesteuropäiscben Völkern behandelt. An dem Beispiel der französischen Revolution im Ausgang des achtzehnten Jahrhunderts wird ihr Einflub bei einem weltgeschichtlichen Ereignis nachgewiesen.

\section{Die Sehnenüberpflanzung}

und ihre Verwertung in der Behandlung der Lähmungen.

Von

Dr. med. Oscar Vulpius,

Professor an der Unirersitžt" Heidelberz.

Wit zahlreichen Figuren and Abbildungen im Text.

gr. 8. 1902. geh. 6 .4. 


\section{Lehrbuch der Speziellen Chirurgie von}

\section{Hermann Tillmanns.}

(Des „Lehrbuches der sllgemeinen und speziellen Chirurgie“ zweiter Band.) Achte, verbesserte and vermehrte Auflage.

\section{Zwei Teile.}

Hit 1207 zum Toll farbigen Abbildungen Im Text.

Roy. 8. geh. $38 \%$, geb. in Halbfranz $43 \%$. 


\section{ALLGEMEINE CHIRURGIE}




\title{
LEHRBUCH
}

DER

\section{A LLGEMEINEN UND SPEZIELLEN \\ CHIRUR G I E}

EINSCHLIESSLICH DER

\section{MODERNEN OPERATIONS- UND VERBANDLEHRE.}

Vow

Dr. HeRmanN TILLMANNS,

GEHEIMER MEDIZINALRAT UND PROFESSOR AN DER UNIVERSITÄT LEIPZIG, GENERALARZT À LA SUITE DES KONIGLICH SÄCHSIBCHEN 8ANITÄTSKORPS.

\author{
ERSTER BAND. \\ LEHRBUCH DER ALLGEMEINEN CHIRURGIE. \\ ALLGEMEINE OPERATIONS- UND VERBAND-TECHNIK. ALLGEMEINE \\ PATHOLOGIE UND THERAPIE.
}

ZEHNTE, VERBESGERTE UND VERMEFRTE AUFLAGE.

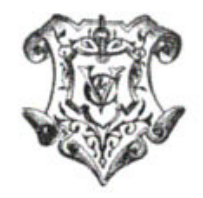

LEIPZIG

VERLAG VON VEIT \& COMP. 


\section{LEHRBUCH}

DER

\section{ALLGEMEINEN CHIRURGIE.}

ALLGEMEINE OPERATIONS- UND VERBAND-TECHNIK. ALLGEMEINE PATHOLOGIE UND THERAPIE.

Vox

DR. HERMANN TILLMANNS,

GEHEIMER MEDIZINALRAT UND PROFESSOR AN DER UNIVERSITÄT LEIPZIG, GENERALARZT Ȧ LA SUITE DES KONIGLICH SÄCHSISCHEN SANITÄTBKORPS.

ZEHNTE, VGRBFBGFRTE UND VFRMFHRTE AUFLAGE.

MIT 787 ZUM TEIL FARBIGEN ABBILDUNGEN IM TEXT.

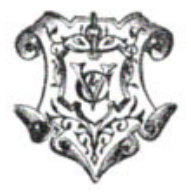

LEIPZIG

VERLAG VON VEIT \& COMP. 
Druck von Metzger \& Wittig in Leipzig. 